\title{
Validation of a parent-proxy quality of life questionnaire for paediatric chronic cough (PC-0OL)
}

\author{
Peter A Newcombe, ${ }^{1,2}$ Jeanie K Sheffield, ${ }^{2}$ Elizabeth F Juniper, ${ }^{3}$ Helen L Petsky, ${ }^{4}$ \\ Carol Willis, ${ }^{4}$ Anne B Chang ${ }^{4,5}$
}

- Additional table and figures are published online only. To view these files please visit the journal online (http://thorax.bmj. com)

${ }^{1}$ School of Social Work and Applied Human Sciences, University of Queensland, Brisbane, Australia ${ }^{2}$ School of Psychology, University of Queensland, Brisbane, Australia ${ }^{3}$ OOL Tech, UK

${ }^{4}$ Queensland Children's Respiratory Centre and Queensland Children's Medical Research Institute, Royal Children's Hospital, Brisbane, Queensland, Australia

${ }^{5}$ Child Health Division, Menzies School of Health Research, Darwin, Northern Territory, Australia

\section{Correspondence to}

Dr Peter Newcombe, School of Social Work and Applied Human Sciences. School of Psychology, The University of Queensland, 11 Salisbury Road, Ipswich, Queensland 4305, Australia; newc@psy.uq.edu.au

Received 20 December 2009 Accepted 26 May 2010

\section{ABSTRACT \\ Background Quality of life (0OL) measures are an important patient-relevant outcome measure for clinical studies. Currently there is no fully validated cough-specific 00L measure for paediatrics. The objective of this study was to validate a cough-specific OOL questionnaire for paediatric use.}

Method 43 children (28 males, 15 females; median age 29 months, IQR 20-41 months) newly referred for chronic cough participated. One parent of each child completed the 27-item Parent Cough-Specific 00L questionnaire (PC-OOL), and the generic child (Pediatric OOL Inventory 4.0 (PedsOL)) and parent OOL questionnaires (SF-12) and two cough-related measures (visual analogue score and verbal category descriptive score) on two occasions separated by 2-3 weeks. Cough counts were also objectively measured on both occasions.

Results Internal consistency for both the domains and total PC-00L at both test times was excellent (Cronbach alpha range $0.70-0.97)$. Evidence for repeatability and criterion validity was established, with significant correlations over time and significant relationships with the cough measures. The PC-0OL was sensitive to change across the test times and these changes were significantly related to changes in cough measures (PC-OOL with: verbal category descriptive score, $r_{s}=-0.37, p=0.016$; visual analogue score, $r_{s}=-0.47$, $p=0.003$ ). Significant correlations of the difference scores for the social domain of the PC-OOL and the domain and total scores of the PedsQL were also noted $\left(r_{s}=0.46, p=0.034\right)$

Conclusion The PC-0OL is a reliable and valid outcome measure that assesses $\mathrm{OOL}$ related to childhood cough at a given time point and measures changes in cough-specific 0OL over time.

\section{INTRODUCTION}

Cough is the most common symptom of respiratory illness, and the majority of children with isolated chronic cough do not have asthma. ${ }^{12}$ It is thus not surprising that cough is a commonly used outcome measure in research and clinical studies. ${ }^{3-5}$ Chronic cough is related to a variety of aetiologies ${ }^{6}$ and may not be categorised into a specific disease process rendering quality of life (QOL) measures for respiratory diseases such as that for asthma unsuitable substitutes for cough research. Disease-specific QOL inventories have been shown to be significantly superior to generic QOL inventories, ${ }^{7}$ with coughspecific QOL measures for adults ${ }^{89}$ showing specificity and sensitivity over generic QOL measures. The inclusion of factors such as urinary incontinence in these adult cough QOL questionnaires, however makes them clearly inappropriate for paediatrics. Furthermore the importance and relevance of paediatric-specific QOL instruments has been increasingly recognised. ${ }^{10-12}$ The adult coughspecific QOL questionnaires, ${ }^{8} 913$ however, highlighted the need for a cough-specific QOL measure and demonstrated the poor appreciation by physicians of the impact of chronic cough on their patients. ${ }^{89}$ Our previous studies have confirmed the high burden of parental concern in children with chronic cough. ${ }^{14} 15$ We have also shown that our preliminary QOL questionnaire derived from a clinical impact method was internally consistent and valid. $^{15}$

As young children are unable to verbally express themselves adequately, it is standard practice for parents to be proxy assessors of their young child's medical condition. ${ }^{16}$ Also, parents/carers are themselves affected by their child's medical condition and thus the parents' own QOL is relevant. Thus, QOL measures used in paediatrics concern the parents/carers themselves (such as in the Short Form Health Survey, SF-12 ${ }^{17}$ ) or are proxy OOL measures (ie, parental perception of their child's illness; eg, Pediatric QOL Inventory 4.0 (PedsOL) ${ }^{18}$ ). A third type of QOL measure for use with children combines both, whereby the items include both parental-proxy and parent's QOL measures such as in Juniper's widely used child asthma OOL questionnaire for carers. ${ }^{16}$

Valid and clinically relevant outcome measures are essential for clinical research. Current outcome measures for paediatric cough are limited. ${ }^{2}$ Indeed we described the first validated paediatric subjective cough system. ${ }^{19}$ The limitations of subjective cough symptom reporting as an isolated symptom has led to groups emphasising the necessity for objective monitoring of cough. ${ }^{20}$ Ambulatory objective cough monitoring systems ${ }^{21}$ that record the number of coughs are available but are limited to research. ${ }^{4}$ The relationships between subjective and objective determinants of cough in children are modest, and the choice of the cough indices depends on the reason for performing the measurement. ${ }^{4}$ Thus, although objective cough measurement is the "gold standard', it is arguably not patient-relevant, necessitating a cough-specific OOL questionnaire as a patient-relevant outcome measure.

There is currently no fully validated cough QOL questionnaire for paediatric use. In this study we aimed to validate our Parent Cough-Specific OOL questionnaire (PC-QOL) ${ }^{15}$ by comparing the questionnaire derived from the clinical impact method with objective and subjective paediatric cough 
measures as well as with generic QOL measures. The sensitivity of the PC-QOL to change over time will also be contrasted to changes in cough measures.

\section{METHODS}

\section{Participants}

Children aged $<14$ years newly referred with chronic cough $\left(>4\right.$ weeks $\left.^{6}\right)$ to the Royal Children's Hospital, Brisbane were eligible. All had a medical intervention that consisted of a consultation and a variety of investigations and treatments depending on the child's clinical history and examination. ${ }^{6}$ Exclusion criteria were: presence of previously diagnosed respiratory diseases (eg, cystic fibrosis), classical asthma (recurrent wheeze or dyspnoea responsive to $\beta_{2}$-agonist) or other underlying disorders such as neurodevelopmental or congenital heart disease. Written consent was obtained from parents and the study was approved by the hospital's human ethics committee.

\section{Procedure}

At initial appointment (Time-1, T1), each parent completed the PC-QOL along with two measures aimed to quantify the chronicity of their child's cough - a cough visual analogue scale (cough VAS) $^{19}$ and a cough verbal category descriptive scale (cough VCD). ${ }^{19}$ The PC-QOL was interviewer administered. Additionally parents completed the PedsOL ${ }^{18}$ and SF-12. ${ }^{17}$ Clinical data were collected on a standardised sheet and parents were given cough diary cards (VAS and VCD) which they completed at home. A digital voice recorder was attached to the child using a lanyard. At a follow-up visit (Time-2, T2) 2-3 weeks postinitial visit, parents repeated all the questionnaires, and ambulatory cough monitoring was re-recorded.

\section{Materials}

\section{Cough measures}

For the cough VAS, parents rated how troublesome their child's cough was on a 10-point scale from $1=$ no trouble to $10=$ troublesome all the time. Child's severity of cough (VCD) was rated on a 6 -point scale $(0=$ no cough to $5=$ severe cough and cannot perform activities), with increasing scores reflecting greater interference with usual activities. This rating (a cough scoring diary) has been validated previously against an objective cough meter measure, and changes in this subjective cough rating have been shown to reflect changes in cough counts. ${ }^{19}$ The digital voice recorder (Sony PCP330F, Japan) recorded continuously for $24 \mathrm{~h}$. The wav file was downloaded, coded and replayed whereby the cough counts (number of coughs) were manually counted by a person blind to all the child's data. As some children did not have $24 \mathrm{~h}$ of recording, cough counts were analysed as number of coughs per hour.

\section{The PC- $00 L^{15}$}

The PC-QOL is a 27-item questionnaire designed to assess the level of frequency of parents' feelings (15 items) and worry (12 items) related to their child's cough. It uses a 7-point Likert-type scale, with higher scores reflecting lower frequency and fewer worry concerns (ie, greater QOL). Previous research ${ }^{15}$ indicates that this 'clinical impact'-derived PC-OOL is both reliable and valid.

\section{The SF-12 v2 ${ }^{17}$}

The SF-12 is a measure of health functioning that computes to eight subscales and two summary scales (Physical Component Summary and Mental Component Summary), with higher scores reflecting better health states.

\section{The PedsOL ${ }^{18}$}

This generic multidimensional questionnaire is designed for parental reports of their child's QOL. It has separate versions for each of four age groups (2-4, 5-7, 8-12 and 13-18 years) and has been used to validate disease-specific and symptom-specific QOL. ${ }^{16}$ The 23 items are responded to on a 5-point Likert-type scale $(0=$ never a problem to $4=$ almost always a problem) and load on to four dimensions of 'functioning' (Physical, Emotional, Social and School Functioning). These can then be grouped into three summary scores (Psychosocial Health, Physical Health and Total). Items were reverse-scored so that higher scores reflected more positive functioning.

\section{Domain allocation}

The clinical impact-derived PC-QOL ${ }^{15}$ formed the basis of the following analyses. The 27-item instrument was first subjected to domain allocation. The domains-psychological, physical and social-were based on the WHO definition of health, ${ }^{22}$ and expert opinion from respiratory paediatricians and mental health professionals. Allocation of items to domains followed a two-step procedure: each item's face validity as determined by the expert panel and each item's correlation with the domain score.

\section{Statistical analysis}

Psychometric properties of reliability (internal consistencyCronbach's alpha, test-retest and consistency-intraclass correlations) and validity (criterion) were examined for the full scale and for each of the domains (see supplementary file online). Further analyses were conducted to determine whether the instrument was sensitive to change over time following medical interventions. Non-parametric analyses (Spearman correlations, Mann-Whitney $U$ test and Wilcoxon signed ranked test) are reported throughout as statistical assumptions essential for parametric analyses were violated (eg, non-normal distributions). Effect sizes are also included. These measure the relative strength of the relationship for the tests of differences in scores across T1 and T2 and quantify the size of this difference over time as a standardised, scale-free measure. Inspection of bivariate scatterplots revealed a number of statistical outliers (see supplementary file). These cases $(n=7)$ depicted an unusual relationship between PC-QOL scores and cough counts/h, and were thus removed from those analyses. Data were analysed using SPSS software v17 (SPSS, Chicago, Illinois, USA).

\section{RESULTS}

Forty-three children (28 males, 15 females; median age 29 months, IOR 20-41 months) and a parent of each child participated in this study. Protracted bacterial bronchitis was diagnosed in 30 children $(69.8 \%)$, bronchiectasis in $6(14 \%)$, asthma in $6(14 \%)$ and tracheomalacia in 1 (2.2\%). Preliminary analyses revealed that PC-OOL total and domain scores did not differ at either T1 or T2 for the participants with protracted bacterial bronchitis compared with those with other chronic respiratory conditions, Mann-Whitney $U$ tests, all $Z$ scores $\leq 1.18$, all $\mathrm{p}$ values $\geq 0.239$. The data across the differing conditions could thus be reliably combined.

The allocation of items to domains identified 11 items in each of the psychological and physical domains and 5 items in the social domain. Cronbach alphas for each of the domains were high (at T1, $\alpha$ range $=0.70-0.90$; at $\mathrm{T} 2, \alpha$ range $=0.86-0.95$ ) and correlations of items with their domains were significant (at T1, range $=0.32-0.81$; at T2, range $=0.53-0.88)$. Cronbach alphas for the full-scale PC-QOL at T1 $(\alpha=0.94)$ and T2 $(\alpha=0.97)$ were 
Table 1 Correlations of domain and total PC-00L scores with cough measures, SF-12 component scores and PedsOL summary scores at initial (T1) and postinitial (T2) visits

\begin{tabular}{|c|c|c|c|c|c|c|c|c|}
\hline & \multicolumn{8}{|l|}{ PC-0OL } \\
\hline & \multicolumn{2}{|l|}{ Psychological } & \multicolumn{2}{|l|}{ Physical } & \multicolumn{2}{|l|}{ Social } & \multicolumn{2}{|l|}{ Total } \\
\hline & $\overline{\mathrm{T} 1}$ & T2 & T1 & T2 & $\overline{\mathrm{T} 1}$ & T2 & $\overline{\mathrm{T} 1}$ & T2 \\
\hline Cough count & $-0.10(0.521)$ & $-0.28(0.089)$ & $-0.21(0.188)$ & $-0.46(0.003)$ & $-0.11(0.487)$ & $-0.51(0.001)$ & $-0.15(0.329)$ & $-0.42(0.008)$ \\
\hline Cough VCD & $-0.36(0.019)$ & $-0.44(0.004)$ & $-0.60(0.001)$ & $-0.42(0.006)$ & $-0.42(0.005)$ & $-0.43(0.005)$ & $-0.49(0.001)$ & $-0.48(0.001)$ \\
\hline Cough VAS & $-0.46(0.003)$ & $-0.59(0.001)$ & $-0.44(0.004)$ & $-0.60(0.001)$ & $-0.33(0.033)$ & $-0.62(0.001)$ & $-0.45(0.003)$ & $-0.67(0.001)$ \\
\hline Mental & $0.31(0.059)$ & $0.33(0.038)$ & $0.23(0.158)$ & $0.20(0.224)$ & $0.27(0.102)$ & $0.11(0.515)$ & $0.30(0.068)$ & $0.24(0.147)$ \\
\hline Total & $0.22(0.171)$ & $0.33(0.042)$ & $0.22(0.188)$ & $0.32(0.045)$ & $0.33(0.038)$ & $0.22(0.180)$ & $0.23(0.163)$ & $0.33(0.038)$ \\
\hline \multicolumn{9}{|l|}{ PedsOL } \\
\hline Psychosocial & $0.38(0.078)$ & $0.21(0.346)$ & $0.21(0.349)$ & $0.31(0.152)$ & $0.31(0.162)$ & $0.41(0.051)$ & $0.30(0.170)$ & $0.33(0.121)$ \\
\hline Physical & $0.56(0.007)$ & $0.39(0.063)$ & $0.58(0.005)$ & $0.45(0.032)$ & $0.32(0.153)$ & $0.62(0.002)$ & $0.50(0.019)$ & $0.48(0.021)$ \\
\hline Total & $0.49(0.019)$ & $0.34(0.108)$ & $0.40(0.063)$ & $0.44(0.036)$ & $0.34(0.128)$ & $0.57(0.004)$ & $0.43(0.048)$ & $0.47(0.024)$ \\
\hline
\end{tabular}

Higher scores on PC-0OL reflect better quality of life; higher scores on cough severity represent greater interference with usual activities; higher scores on cough VAS represent greater trouble with the cough; higher scores on SF-12 reflect better health states; and higher scores on PedsOL reflect more positive functioning.

Numbers represent Spearman correlations with probability levels in parentheses.

PC-0OL, Parent Cough-Specific 0OL questionnaire; PedsOL, Pediatric OOL Inventory 4.0; SF-12, Short Form Health Survey-12; VAS, verbal analogue scale; VCD, verbal category descriptive scale.

both excellent. Test-retest reliability analyses revealed intraclass correlations for the domains of the PC-QOL and the full scale to be high and significant, ranging from 0.40 to 0.51 , all $\mathrm{p}$ values $\leq 0.004$. Intraclass correlations for the cough measures were significant for both cough counts $/ \mathrm{h}\left(\mathrm{r}_{\mathrm{s}}=0.43, \mathrm{p}<0.003\right)$ and cough VAS $\left(r_{s}=0.36, p=0.013\right)$, but not for cough VCD $\left(r_{s}=0.20\right.$, $\mathrm{p}=0.100)$.

Evidence for criterion validity was examined through the relationships between scores on the PC-QOL and the cough measures. The correlations for T1 and T2 are presented in table 1 and, for the most part, support criterion validity. There were significant correlations for the domains and total PC-QOL and VCD and VAS across all measures and time points and for the cough counts/h at postinitial visit for the social domain and total score. The correlations of the PC-OOL with SF-12 and PedsOL revealed some significant and some high absolute relationships especially with the total component score of the SF-12 at T2 and the physical summary and total scores of the PedsQL. A low completion rate of the PedsOL $(n=23)$ is a likely explanation for the few significant correlations despite satisfactory absolute values. This low completion rate was due to an administrative error and not reflected in the completion of other instruments.

Evidence was also gained by examining the correlations between the difference scores-that is, whether the difference score in $\mathrm{PC}-\mathrm{QOL}$ across the two test times was related to difference scores in the cough measures. Total PC-QOL correlated significantly with cough VCD score $\left(r_{s}=-0.55, p=0.001\right)$ and cough VAS $\left(r_{s}=-0.55, p=0.001\right)$, with a trend to significance for cough counts $/ h\left(r_{s}=-0.32, p=0.074\right)$. Significant correlations of the difference scores for the social domain of PC-QOL and the domains and total PedsQL were also noted, $r_{s} \geq 0.46, p \leq 0.034$. Further, the PC-QOL was, as were the cough measures, sensitive to change over time, Wilcoxon ranked sign tests, Z scores $\geq 3.01$, $p$ values $\leq 0.003$. The means for each measure across time are presented in figure 1. The effect sizes for change in domain and total PC-QOL scores and cough measures are presented in table 2.

\section{DISCUSSION}

We validated our PC-OOL derived from the clinical impact method by comparing PC-OOL with other measures of cough severity (subjective cough scores, objective cough counts) and generic QOLs (PedsQL and SF-12) across two time frames in 43 newly referred children with chronic cough. We found that the PC-QOL demonstrated high levels of both internal consistency and test-retest consistency-that is, the items all reliably loaded on a single construct of paediatric cough-related $\mathrm{OOL}$ and measurement of this construct was consistent over a 2-3 week period. High internal consistencies for the full-scale PC-QOL may represent possible redundancy of items; however, closer inspection revealed that all items added significantly to the construct in terms of both face validity and statistical estimates. Criterion validity of the PC-QOL was endorsed, with significant correlations of domains and total scores with the component and summary scores of the SF-12 and PedsQL. We also found that PCQOL scores were sensitive to change over time, and mirrored the changes in other cough severity measures (subjective and objective). The findings from the present study endorse the 27-item $\mathrm{PC}-\mathrm{QOL}$ as a cough-specific QOL measure that is reliable and valid.

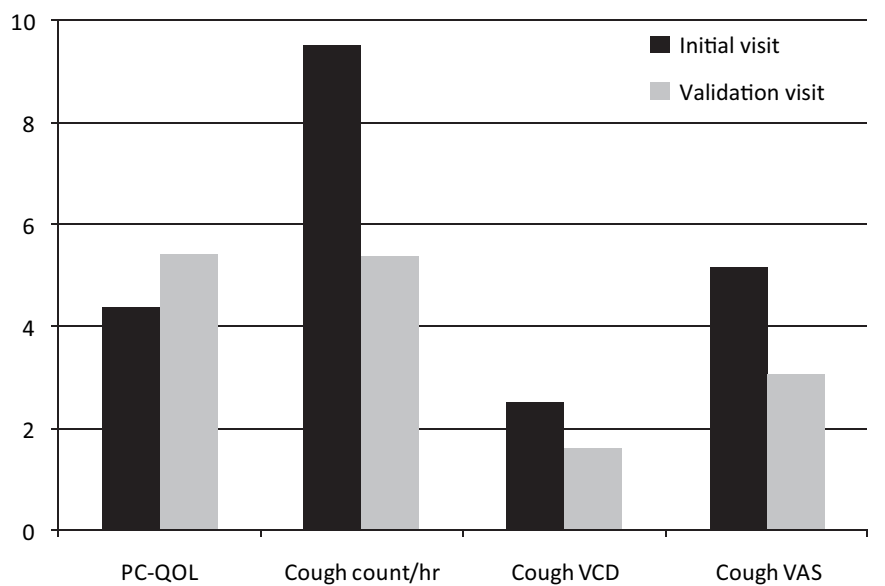

Figure 1 Mean scores across time for the total Parent Cough-Specific $00 \mathrm{~L}$ questionnaire (PC-0OL) and the cough measures of cough counts $/ \mathrm{h}$, cough verbal category descriptive scale (VCD) and cough visual analogue scale (VAS). Higher scores on the PC-00L reflect better quality of life; higher scores on the cough VCD represent greater interference with usual activities; higher scores on the cough VAS represent greater trouble with the cough. Cough counts were objectively measured. 
Table 2 Effect sizes for differences in PC-0OL and cough measure responsiveness to change over time

\begin{tabular}{lc}
\hline Measure & Effect size \\
\hline PC-00L & \\
Psychological & 0.32 \\
Physical & 0.41 \\
Social & 0.32 \\
Total & 0.39 \\
Cough count/h (objectively measured) & 0.19 \\
Cough VCD & 0.31 \\
Cough VAS & 0.43 \\
\hline PC-OOL, Parent Cough-Specific OOL questionnaire; VAS, verbal analogue \\
scale; VCD, verbal category descriptive scale.
\end{tabular}

Adult cough-specific QOL questionnaires have been used for several years. ${ }^{8}$ Similar to the Leicester Cough Questionnaire, ${ }^{9}$ our PC-QOL covers three domains highlighted by the WHO definition of health, was derived from a larger pool of items by the clinical impact method ${ }^{16}$ and was validated against measures of generic QOL measures. An important difference, however, is that we have determined the PC-OOL validity against both objective (cough counts) and subjective (VCD, VAS) measures of cough severity. Our questionnaire is also longer (27 items compared with 19 items) but covers both worry and frequency of parent's concerns for the effect of their child's chronic cough. Compared with the Cough Quality of Life Questionnaire, ${ }^{8}$ the PC-QOL is of similar length but covers fewer domains. Although the Cough Quality of Life Questionnaire demonstrated strong internal and test-retest reliability, information about its criterion validity and its sensitivity to change over time was not reported. ${ }^{8}$

In this study we present the first validated cough-specific QOL questionnaire for paediatric use. It has corroborated our previous findings ${ }^{15}$ of high reliability and strong validity in an instrument designed to identify and locate areas of concern for parents and carers of children with chronic cough. Validity was confirmed, with strong relationships across two time points to both subjective cough measures and objective cough counts. The support for the measure was further enhanced by the significant comparisons with a previously validated measure of generic adult QOL (SF-12) and a generic multidimensional paediatric $\mathrm{QOL}$ (PedsOL) instrument. In the validation process, it is important to show that a measure can be sensitive to change and that this sensitivity emulates changes in correspondingly validated measures. Our instrument was able to detect changes in QOL following a clinical intervention over $2-3$ weeks and these changes were associated with observed changes in cough measures and counts, as well as changes in PedsOL.

The item stems in our PC-QOL capture frequency and worry about aspects of child and family functioning affected by a child's chronic cough. This is similar to the widely used asthma POLO developed by Juniper et al. ${ }^{16}$ The items in our PCQOL can be reliably partitioned into the domains of psychological, social and physical concerns, as well as a total measure of OOL. The WHO domains of psychological, physical and social functioning were confirmed through face validity supported by expert opinion. The domains were also shown to be sensitive to change following clinical intervention.

One limitation of the present study was the relatively short time period between testing times. A longer time interval would enable a more thorough examination of the sensitivity of the instrument to detect change. Although there were some significant correlations to the SF-12 and PedsOL at both testing times and across time, these were not consistent. It may be that the
PC-QOL and its domains are measuring slightly different aspects of functioning compared with the SF-12 and PedsOL. SF-12 specifically assesses the parent's QOL (as opposed to the child's) and PedsOL draws on more generalised functioning (as opposed to specific illness related). Thus further research in this area is warranted as the relatively small sample sizes for some of the analyses limits the generalisability of the results. Also, in the present study, the items were researcher administered for greater control. However, we believe that the PC-QOL can be self-administered and, under these conditions, would take $10-15 \mathrm{~min}$ to complete. An extension of a childrenspecific cough $\mathrm{QOL}$ questionnaire that is child completed is also required. Also, the minimal important difference for our $\mathrm{PC}-\mathrm{QOL}$ is yet to be determined.

The clinical utility of the PC-QOL is readily apparent as an outcome measure for evaluating interventions in clinical research involving children with cough. Furthermore, its impact can stretch to identifying foci for non-medical interventions to accompany medical interventions. In this regard, it can provide insight into the impact of treatment across aspects of life that may be related to a child's chronic cough condition but can sometimes be ignored due to a focus on the illness component of treatment. In particular, the PC-OOL can assist in identifying potential psychological, social and physical areas requiring assistance. It may also be particularly useful to assist clinicians in assessing whether clinical interventions have a significant and positive impact on both physical and psychosocial functioning over time.

The PC-QOL can be completed quickly and easily by parents, and could be included as part of the baseline assessment of patients as well as for evaluation and monitoring of treatment for cough-dominant conditions. The strong psychometric features of the measure and its significant correlation with other QOL measures and subjective and objective measures of cough support its robustness as a valid outcome measure. The performance of PC-QOL further reinforces the importance and relevance of paediatric-specific $\mathrm{QOL}$ instruments for this population. ${ }^{10-12}$

Acknowledgements We thank the parents and children who participated in the study. We also thank Drs Masters, Francis and Dore for allowing their patients to participate in the study.

Funding The study was supported by a Royal Children's Hospital Foundation Grant (Brisbane, Australia). AC is supported by an NHMRC Practitioner Fellowship (Grant \#545216).

\section{Competing interests None.}

Ethics approval This study was conducted with the approval of the Royal Children's Hospital Human Ethics Committee, Brisbane, Australia.

Provenance and peer review Not commissioned; externally peer reviewed.

\section{REFERENCES}

1. Chang AB, Phelan PD, Carlin J, et al. Randomised controlled trial of inhaled salbutamol and beclomethasone for recurrent cough. Arch Dis Child 1998:79:6-11.

2. Chang AB. Cough: are children really different to adults? Cough 2005;1:7.

3. Andriessen JW, Brunekreef B, Roemer W. Home dampness and respiratory health status in European children. Clin Exp Allergy 1998;28:1191-200.

4. Chang AB, Phelan PD, Robertson CF, et al. Relationship between measurements of cough severity. Arch Dis Child 2003;88:57-60.

5. Murray MP, Turnbull K, Macquarrie S, et al. Validation of the Leicester Cough Questionnaire in non-cystic fibrosis bronchiectasis. Eur Respir J 2009;34:125-31.

6. Chang $\mathbf{A B}$, Landau $\mathrm{LI}$, van Asperen PP, et al. Cough in children: definitions and clinical evaluation. Med J Aust 2006;184:398-403.

7. Wiebe S, Guyatt G, Weaver B, et al. Comparative responsiveness of generic and specific quality-of-life instruments. J Clin Epidemiol 2003;56:52-60. 
8. French CT, Irwin RS, Fletcher KE, et al. Evaluation of a cough-specific quality-of-life questionnaire. Chest 2002;121:1123-31.

9. Birring SS, Prudon B, Carr AJ, et al. Development of a symptom specific health status measure for patients with chronic cough: Leicester Cough Questionnaire (LCQ). Thorax 2003;58:339-43.

10. Powers SW, Patton SR, Hommel KA, et al. Quality of life in childhood migraines: clinical impact and comparison to other chronic illnesses. Pediatrics 2003;112:e1-5.

11. Mansour ME, Kotagal U, Rose B, et al. Health-related quality of life in urban elementary schoolchildren. Pediatrics 2003;111:1372-81.

12. Landgraf JM, Abetz L. Measuring health outcomes in pediatric populations: issues in psychometrics and application. In: Spilker B, ed. Quality of Life and Pharmacoeconomics in Clinical Trials. Philadelphia: Lippincott-Raven Publishers, 2004:793-802.

13. Polley L, Yaman N, Heaney L, et al. Impact of cough across different chronic respiratory diseases: comparison of two cough-specific health-related quality of life questionnaires. Chest 2008;134:295-302.

14. Marchant JM, Newcombe PA, Juniper EF, et al. What is the burden of chronic cough for families? Chest 2008;134:303-9.
15. Newcombe PA, Sheffield JK, Juniper EF, et al. Development of a parent-proxy quality-of-life chronic cough-specific questionnaire: clinical impact vs psychometric evaluations. Chest 2008;133:386-95.

16. Juniper EF, Guyatt $\mathrm{GH}$, Feeny $\mathrm{DH}$, et al. Measuring quality of life in children with asthma. Qual Life Res 1996;5:35-46.

17. Ware J Jr, Kosinski M, Keller SD. A 12-Item Short-Form Health Survey: construction of scales and preliminary tests of reliability and validity. Med Care 1996;34:220-33

18. Varni JW, Burwinkle TM, Seid M, et al. The PedsQL 4.0 as a pediatric population health measure: feasibility, reliability, and validity. Ambul Pediatr 2003;3:329-41.

19. Chang AB, Newman RG, Carlin J, et al. Subjective scoring of cough in children: parent-completed vs child-completed diary cards vs an objective method. Eur Respir J 1998;11:462-6.

20. de Blic J, Thomson A. Short-term clinical measurement: acute severe episodes Eur Respir J Supp/ 1996:21:4s-7s.

21. Chang AB, Newman RG, Phelan PD, et al. A new use for an old Holter monitor: an ambulatory cough meter. Eur Respir J 1997;10:1637-9.

22. World Health Organization. Constitution of the World Health Organization. Geneva: WHO, 1947.

\section{Lung alert}

\section{Immunoglobulin G2 subclass deficiency and H1N1}

Severe $\mathrm{H} 1 \mathrm{~N} 1$ infection is associated with risk factors that include pregnancy, obesity and immunosuppression. This study assessed levels of serum immunoglobulin G (IgG) and IgG subclasses in patients with severe H1N1 infection (defined as infection requiring respiratory and/or vasopressor support) and moderate $\mathrm{H} 1 \mathrm{~N} 1$ infection (defined as those requiring hospitalisation) and in healthy pregnant women.

Thirty-nine patients (19 with severe infection and 20 with moderate infection) were assessed. The presence of severe H1N1 infection was significantly associated with low levels of total IgG, IgG1 and IgG2 compared with moderate infection, and similarly hypoalbuminaemia and anaemia, but only hypoalbuminaemia and low mean IgG2 levels remained statistically significant after multivariate analysis. Convalescent phase serum samples were obtained from 15 IgG2-deficient patients (11 with severe infection and 4 with moderate infection) at a mean of $90 \pm 23$ days. Eight $(73 \%)$ patients with severe infection remained IgG2 deficient, but hypoalbuminaemia was resolved in most patients. Pregnant women with $\mathrm{H} 1 \mathrm{~N} 1$ infection had significantly lower mean levels of IgG2 compared with the pregnant control subjects.

Severe H1N1 infection is associated with IgG2 deficiency, which appears to persist, but it is uncertain whether those patients had underlying IgG2 deficiency, or whether interaction between H1IN1 infection and the host B cells leads to such deficiency. Nevertheless, this may prove an interesting therapeutic avenue to pursue in the future.

Gordon CL, Johnson PDR, Permezel M, et al. Association between severe pandemic 2009 influenza A (H1N1) virus infection and immunoglobulin G2 subclass deficiency. Clin Infect Dis 2010;50:673-8.

\section{B Kathiresan}

Correspondence to Bhuvaneswari Kathiresan, Plymouth Hospitals NHS Trust, Plymouth, UK; doctorbhuvaneshwarii@rediffmail.com

Thorax 2010;65:823. doi:10.1136/thx.2010.145649 